\title{
Effect of surface stresses on surface waves in elastic solids
}

\author{
PRANABES KANTI PAL ${ }^{1}$, D ACHARYA ${ }^{2}$ and P R SENGUPTA ${ }^{3}$ \\ ${ }^{1}$ Indian Institute of Mechanics of Continua, 201, Manicktola Main Road, \\ Flat no 42, Calcutta 700054 , India \\ ${ }^{2}$ Department of Mathematics, Mahadevananda College, Burrackpore 743 101, \\ India \\ ${ }^{3}$ Department of Mathematics, University of Kalyani, Kalyani 741235 , India
}

MS received 25 July 1996; revised 19 July 1997

\begin{abstract}
This paper deals with the propagation of surface waves in homogeneous, elastic solid media whose free surfaces or interfaces of separation are capable of supporting their own stress fields. The general theory for the propagation of surface waves in a medium which supports surface stresses is first deduced, and then this theory is employed to investigate the particular cases of surface waves, viz. (a) Rayleigh waves, (b) Love waves and (c) Stoneley waves. It is seen that the Rayleigh waves become dispersive in nature; and, in case of low frequency with residual surface tension, a critical wavelength exists, below which the propagation of Rayleigh waves is not possible. This critical wave length is directly proportional to the surface tension. Some numerical calculations have been made in the case of Love waves and conclusions have been drawn.
\end{abstract}

Keywords. Surface waves; effect of surface stresses; Rayleigh waves; Love waves; Stoneley waves; residual stress.

\section{Introduction}

Surface waves play an important role in the science of earthquakes. To match the actual situation in a given problem, three types of surface waves are generally introduced which are classified according to their nature of displacements (Dey \& Sengupta 1978; Chandrasekharaiah 1986; Das et al 1992; Pal et al 1996). Again it is known that the physical properties of bodies in the neighbourhood of the surface are sensibly different from those in the interior. Thus the boundary surface may be regarded as a two-dimensional elastic continuum which adheres to the body without slipping (Gurtin \& Murdoch 1976; Chandrasekharaiah 1987). Different authors (Plaster 1972; Chandrasekharaiah 1987) presented their research papers including surface stresses as their subject of discussion. In the 
present paper, following a theory of surface stresses, the authors investigate surface wave propagation. The Rayleigh wave velocity equation has been obtained as a particular case. Following Chandrasekharaiah (1987), the effect of surface stresses on Rayleigh wave propagation has been discussed. Next we discuss Love wave propagation with surface stress on its free boundry, with some numerical computations and conclusions. Wave velocity equation for Stoneley waves has also been deduced.

\section{Basic equations}

Let us consider two homogeneous, isotropic, elastic solid media $M_{1}$ (lower medium) and $M_{2}$ (upper medium) with different material constants. We also consider that a twodimensional elastic layer is present at the surface of separation of the two media. We take an orthogonal cartesian frame of axes $O x_{1} x_{2} x_{3}, O$ is taken on the interface and $O x_{3}$ is positive in the downward direction. As in Bullen \& Bolt (1985), here also the wave travels in the $O x_{1}$ direction. The disturbances of the particles on lines parallel to $O x_{2}$ vibrate in phase, which means all partial derivatives with respect to $x_{2}$ are zero.

The equation of motion in the absence of body forces is

$$
\mu \nabla^{2} \mathbf{u}+(\lambda+\mu) \operatorname{grad} \operatorname{div} \mathbf{u}=\rho \frac{\partial^{2} \mathbf{u}}{\partial t^{2}} .
$$

In the above Navier's equation of classical elasticity $\mathbf{u}\left(u_{1}, u_{2}, u_{3}\right)$ is the displacement vector; $\lambda, \mu$ are Lamé elastic constants; $\rho$ is mass density and $t$ represents time. To investigate plane deformation parallel to the $x_{1} x_{3}$ plane we introduce the displacement potentials $P, Q$ related to the displacement components $u_{1}\left(x_{1}, x_{3}, t\right)$ and $u_{3}\left(x_{1}, x_{3}, t\right)$ by the equations,

$$
u_{1}=\frac{\partial P}{\partial x_{1}}-\frac{\partial Q}{\partial x_{3}}, \quad u_{3}=\frac{\partial P}{\partial x_{3}}+\frac{\partial Q}{\partial x_{1}} .
$$

Introducing (2) in (1) we obtain,

$$
\begin{aligned}
& \left(\nabla^{2}-\frac{1}{a^{2}} \frac{\partial^{2}}{\partial t^{2}}\right) P=0, \\
& \left(\nabla^{2}-\frac{1}{b^{2}} \frac{\partial^{2}}{\partial t^{2}}\right) Q=0, \\
& \left(\nabla^{2}-\frac{1}{b^{2}} \frac{\partial^{2}}{\partial t^{2}}\right) u_{2}=0,
\end{aligned}
$$

where $a=(\lambda+2 \mu / \rho)^{1 / 2}$ and $b=(\mu / \rho)^{1 / 2}$ are velocities of dilatation and distortion respectively. Befitting the actual situation of the problem, we seek solutions to (3)-(5) as

$$
\left[P, Q, u_{2}\right]=\left[\bar{P}\left(x_{3}\right), \bar{Q}\left(x_{3}\right), \bar{u}_{2}\left(x_{3}\right)\right] \mathrm{e}^{i\left(\eta x_{1}-\zeta t\right)}
$$


Inserting (6) in (3)-(5), we get

$$
\begin{aligned}
& \frac{\mathrm{d}^{2} \bar{P}}{\mathrm{~d} x_{3}^{2}}-\eta^{2}\left(1-\frac{\zeta^{2}}{\eta^{2} a^{2}}\right) \bar{P}=0 \\
& \frac{\mathrm{d}^{2} \bar{Q}}{\mathrm{~d} x_{3}^{2}}-\eta^{2}\left(1-\frac{\zeta^{2}}{\eta^{2} b^{2}}\right) \bar{Q}=0, \\
& \frac{\mathrm{d}^{2} \bar{u}_{2}}{\mathrm{~d} x_{3}^{2}}-\eta^{2}\left(1-\frac{\zeta^{2}}{\eta^{2} b^{2}}\right) \bar{u}_{2}=0 .
\end{aligned}
$$

Now, $\bar{P}\left(x_{3}\right), \bar{Q}\left(x_{3}\right), \bar{u}_{2}\left(x_{3}\right)$ describe surface waves and as such they must be vanishingly small as $x_{3} \rightarrow \infty$. Hence the solution of (5) for the medium $M_{1}$ may be taken as

$$
\begin{gathered}
P=A_{1} \exp \left(-\eta m_{1} x_{3}\right) \mathrm{e}^{i\left(\eta x_{1}-\zeta t\right)} . \\
Q=A_{2} \exp \left(-\eta m_{2} x_{3}\right) \mathrm{e}^{i\left(\eta x_{1}-\zeta t\right)}, \\
u_{2}=A_{3} \exp \left(-\eta m_{2} x_{3}\right) \mathrm{e}^{i\left(\eta x_{1}-\zeta t\right)},
\end{gathered}
$$

where

$$
\begin{aligned}
& m_{1}=\left(1-r^{2}\right)^{1 / 2}, m_{2}=\left(1-s^{2}\right)^{1 / 2}, \\
& c=\frac{\zeta}{\eta}, \quad r=\frac{c}{a}, \quad s=\frac{c}{b},
\end{aligned}
$$

wavelength $=2 \pi / \eta$, wave velocity $=\zeta / \eta, \zeta$ being a known constant while $\eta$ is an unknown constant; and, $A_{1}, A_{2}, A_{3}$ are arbitrary constants.

Analogous solutions with primes (for the region $0 \leq x_{3}<-\infty$ ) are

$$
\begin{aligned}
& P^{\prime}=A_{1}^{\prime} \exp \left(\eta m_{1}^{\prime} x_{3}\right) \mathrm{e}^{i\left(\eta x_{1}-\zeta t\right)}, \\
& Q^{\prime}=A_{2}^{\prime} \exp \left(\eta m_{2}^{\prime} x_{3}\right) \mathrm{e}^{i\left(\eta x_{1}-\zeta t\right)}, \\
& u_{2}^{\prime}=A_{3}^{\prime} \exp \left(\eta m_{2}^{\prime} x_{3}\right) \mathrm{e}^{i\left(\eta x_{1}-\zeta t\right)},
\end{aligned}
$$

are obtained for the upper medium $M_{2}$ of the material, where

$$
\begin{aligned}
m_{1}^{\prime} & =\left(1-r^{\prime 2}\right)^{1 / 2}, \quad m_{2}^{\prime}=\left(1-s^{\prime 2}\right)^{1 / 2}, \\
c & =\frac{\zeta}{\eta}, \quad r^{\prime}=\frac{c}{a^{\prime}}, \quad s^{\prime}=\frac{c}{b^{\prime}} .
\end{aligned}
$$

\section{Boundary conditions and solutions}

We assume that the plane $x_{3}=0$ is a material layer which adheres, without slipping, to the lower medium and is a two-dimensional elastic continuum. This layer is capable of supporting its own stress represented by surface stress tensor $\sum_{i \alpha}$, and obeys the law (Chandrasekharaiah 1987),

$$
\sum_{i \alpha}= \begin{cases}\delta_{i \alpha}\left[\sigma+\left(\lambda_{0}+\sigma\right) u_{\gamma, \gamma}\right]+\mu_{0} u_{i, \alpha}+\left(\mu_{0}-\sigma\right) u_{\alpha, i}, & \text { for } i, \alpha, \gamma=1,2 \\ \sigma u_{3, \alpha}, & \text { for } i=3\end{cases}
$$


Here $\lambda_{0}, \mu_{0}$ are the Lamé moduli of the material boundary and $\sigma$ is the residual surface tension on the layer $x_{3}=0$. Following Gurtin \& Murdoch (1976) we use the surface elasticity tensor. The forces on the bounding surface are governed by surface stress tensor $\sum_{i \alpha}$. Given a smooth oriented curve $\gamma$ in $x_{3}=0$ with positive unit normal $n_{i}, \sum_{i \alpha} n_{\alpha}$ is the force per unit length of $\gamma$ by that part of the boundary into which $n_{\alpha}$ is directed, upon that part of $x_{3}=0$ away from which $n_{\alpha}$ points. Dimensions of $\lambda_{0}, \mu_{0}, \sigma$ are the same $(\mathrm{N} / \mathrm{m})$.

The boundary conditions are

$$
\tau_{i 3}+\sum_{i \alpha, \alpha}-\rho_{0} \frac{\partial^{2} u_{i}}{\partial t^{2}}=\tau_{i 3}^{\prime}, \quad \text { on } x_{3}=0,
$$

where $\rho_{0}$ is the mass per unit surface area $\left(\mathrm{kg} \mathrm{m}^{-2}\right)$ of the layer (Gurtin \& Murdoch 1976), and $\tau_{i j}$ and $\tau_{i j}^{\prime}$ are the stress tensors in the interior of the media $M_{1}$ and $M_{2}$ respectively. The conventional stress tensor $\tau_{i 3}$ has the unit of force per unit area and the new stress tensor $\sum_{i \alpha}$ has the unit of force per unit length. $\tau_{i j}$ obeys the law (Gurtin \& Murdoch 1976),

$$
\tau_{i j}=\lambda \delta_{i j} u_{k, k}+\mu\left(u_{i, j}+u_{j, i}\right) .
$$

The boundary conditions are

$$
\begin{aligned}
& \mu\left(2 \frac{\partial^{2} P}{\partial x_{1} \partial x_{3}}+\frac{\partial^{2} Q}{\partial x_{1}^{2}}-\frac{\partial^{2} Q}{\partial x_{3}^{2}}\right)+\left[\left(\lambda_{0}+2 \mu_{0}\right) \frac{\partial^{2}}{\partial x_{1}^{2}}-\rho_{0} \frac{\partial^{2}}{\partial t^{2}}\right]\left(\frac{\partial P}{\partial x_{1}}-\frac{\partial Q}{\partial x_{3}}\right) \\
& \quad=\mu^{\prime}\left(2 \frac{\partial^{2} P^{\prime}}{\partial x_{1} \delta x_{3}}+\frac{\partial^{2} Q^{\prime}}{\partial x_{1}^{2}}-\frac{\partial^{2} Q^{\prime}}{\partial x_{3}^{2}}\right), \\
& 2 \mu\left(\frac{\partial^{2} Q}{\partial x_{1} \partial x_{3}}-\frac{\partial^{2} P}{\partial x_{1}^{2}}\right)+\frac{\mu}{b^{2}} \frac{\partial^{2} P}{\partial t^{2}}+\left(\alpha \frac{\partial^{2}}{\partial x_{1}^{2}}-\rho_{0} \frac{\partial^{2}}{\partial t^{2}}\right)\left(\frac{\partial P}{\partial x_{3}}+\frac{\partial Q}{\partial x_{1}}\right) \\
& \quad=2 \mu^{\prime}\left(\frac{\partial^{2} Q^{\prime}}{\partial x_{1} \partial x_{3}}-\frac{\partial^{2} P^{\prime}}{\partial x_{1}^{2}}\right)+\frac{\mu^{\prime}}{b^{\prime 2}} \frac{\partial^{2} P^{\prime}}{\partial t^{2}}\left(\mu_{0} \frac{\partial^{2}}{\partial x_{1}^{2}}-\rho_{0} \frac{\partial^{2}}{\partial t^{2}}+\mu \frac{\partial}{\partial x_{3}^{2}}\right) u_{2} \\
& =\mu^{\prime} \frac{\partial^{2} u_{2}^{\prime}}{\partial x_{3}^{2}} .
\end{aligned}
$$

(ii) The displacement components must be continuous on the surface of separation $x_{3}=0$ at all places and times. Therefore

$$
\begin{aligned}
& \frac{\partial P}{\partial x_{1}}-\frac{\partial Q}{\partial x_{3}}=\frac{\partial P^{\prime}}{\partial x_{1}}-\frac{\partial Q^{\prime}}{\partial x_{3}} \\
& \frac{\partial P}{\partial x_{3}}+\frac{\partial Q}{\partial x_{1}}=\frac{\partial P^{\prime}}{\partial x_{3}}+\frac{\partial Q^{\prime}}{\partial x_{1}} \\
& u_{2}=u_{2}^{\prime}
\end{aligned}
$$


Applying boundary conditions (14) and (14a) to (8) and (10) the following system of equations is obtained,

$$
\begin{aligned}
& \left(2 m_{1}+\eta F\right) A_{1}-i\left(2-s^{2}+\eta m_{2} F\right) A_{2} \\
& \quad+\frac{2 \mu^{\prime}}{\mu} m_{1}^{\prime} A_{1}^{\prime}+i \frac{\mu^{\prime}}{\mu}\left(2-s^{\prime 2}\right) A_{2}^{\prime}=0 \\
& \left(2-s^{2}+\eta m_{1} H\right) A_{1}-i\left(2 m_{2}+\eta H\right) A_{2} \\
& \quad-\frac{\mu^{\prime}}{\mu}\left(2-s^{\prime 2}\right) A_{1}^{\prime}-i 2 \frac{\mu^{\prime}}{\mu} m_{2}^{\prime} A_{2}^{\prime}=0 \\
& A_{1}-i m_{2} A_{2}-A_{1}^{\prime}-i m_{2}^{\prime} A_{2}^{\prime}=0 \\
& m_{1} A_{1}-i A_{2}+m_{1}^{\prime} A_{1}^{\prime}+i A_{2}^{\prime}=0 \\
& \left(\mu_{0}-\rho_{o} c^{2}+\frac{\mu}{\eta} m_{2}\right) A_{3}+\frac{\mu^{\prime}}{\eta} m_{2}^{\prime} A_{3}^{\prime}=0 \\
& A_{3}-A_{3}^{\prime}=0
\end{aligned}
$$

where we have taken

$$
\begin{aligned}
& \frac{1}{\mu}\left(\lambda_{0}+2 \mu_{0}-\rho_{0} c^{2}\right)=F, \\
& \frac{1}{\mu}\left(\sigma-\rho_{0} c^{2}\right)=H .
\end{aligned}
$$

The last two equations of (15) yield

$$
A_{3}=A_{3}^{\prime}=0 \text {, }
$$

which implies that there is no propagation of displacement $u_{2}$. Eliminating the constants $A_{1}, A_{2}, A_{1}^{\prime}, A_{2}^{\prime}$ from (15) we obtain

$$
\Delta=\operatorname{det}\left[a_{i j}\right]=0, \quad i, j=1,2,3,4,
$$

where

$$
\begin{aligned}
& a_{11}=2 m_{1}+\eta F, a_{12}=2-s^{2}+\eta m_{2} F, \\
& a_{13}=2 \frac{\mu^{\prime}}{\mu} m_{1}^{\prime}, a_{14}=\frac{\mu^{\prime}}{\mu}\left(2-s^{2}\right), \\
& a_{21}=2-s^{2}+\eta m_{1} H, a_{22}=2 m_{2}+\eta H, \\
& a_{23}=-\frac{\mu^{\prime}}{\mu}\left(2-s^{\prime 2}\right), \\
& a_{24}=-2 \frac{\mu^{\prime}}{\mu} m_{2}^{\prime}, \\
& a_{31}=1, a_{32}=m_{2}, a_{33}=-1, a_{34}=-m_{2}^{\prime}, \\
& a_{41}=m_{1}, a_{42}=1, a_{43}=m_{1}^{\prime}, a_{44}=1 .
\end{aligned}
$$

Equation (17) represents the wave velocity dispersion equation for interface waves in elastic solid media under the influence of surface stresses, where the plane of separation 
$x_{3}=0$ is a material boundary. The equation depends on the particular value of $\eta$ which indicates dispersive nature caused by the presence of surface stresses. If the surface of separation is free of surface stresses, $F=H=0$; and at once the classical equation of general surface waves is obtained from (17).

\section{Particular cases}

\subsection{Rayleigh waves}

As a particular case of the general surface wave discussed in the previous article we may study Rayleigh waves under the influence of surface stresses in the light of the investigation done by Chandrasekharaiah (1987). Here the upper medium $\left(M_{2}\right)$ is replaced by vacuum. Applying the boundary conditions

$$
\begin{gathered}
\tau_{13}+\sum_{1 \alpha, \alpha}-\rho_{0} \frac{\partial^{2} u_{1}}{\partial t^{2}}=0, \\
\tau_{33}+\sum_{3 \alpha, \alpha}-\rho_{0} \frac{\partial^{2} u_{3}}{\partial t^{2}}=0,
\end{gathered}
$$

we get

$$
\begin{aligned}
\left(2 m_{1}+\eta F\right) A_{1}+\left(2-s^{2}+\eta m_{2} F\right) A_{2} & =0 \\
\left(2-s^{2}+\eta m_{1} H\right) A_{1}+\left(2 m_{2}+\eta H\right) A_{2} & =0 .
\end{aligned}
$$

Eliminating the constants $A_{1}, A_{2}$ the Rayleigh wave velocity equation may be obtained as

$$
\left|\begin{array}{cc}
2 m_{1}+\eta F & 2-s^{2}+\eta m_{2} H \\
2-s^{2}+\eta m_{1} H & 2 m_{2}+\eta H
\end{array}\right|=0 .
$$

The determinantal equation on simplification gives

$$
\left(1-m_{1} m_{2}\right) F H \eta^{2}+\left(m_{1} H+m_{2} F\right) s^{2} \eta+4 m_{1} m_{2}-\left(2-s^{2}\right)^{2}=0 .
$$

This equation is in agreement with the corresponding equation obtained by Chandrasekharaiah (1987) in the absence of voids. Again in the case of conventional stress-free boundary, (18) becomes

$$
4 m_{1} m_{2}-\left(2-s^{2}\right)^{2}=0 .
$$

When surface stresses $\sum_{i \alpha}$ arise from surface tension, we have as a special case the conditions $\lambda_{0}=\mu_{0}=\rho_{0}=0$. In such a situation $F=0, H=\sigma / \mu$ and hence the equation (18) becomes

$$
\eta \sigma s^{2}\left(1-s^{2} \tau_{0}^{2}\right)^{1 / 2}=\mu\left[\left(2-s^{2}\right)^{2}-4\left(1-s^{2}\right)^{1 / 2}\left(1-s^{2} \tau_{0}^{2}\right)^{1 / 2}\right],
$$


where

$$
\tau_{0}^{2}=b^{2} / a^{2} .
$$

This equation is in agreement with the corresponding equation obtained by Chandrasekharaiah (1987) in the absence of voids. In the absence of residual surface tension, we take $\sigma=0$ to obtain the classical Rayleigh wave velocity equation (19). In this case, (20) has one root $s=s_{0}$ in the open interval $(0,1)$. If $\sigma \neq 0$, (20) has a real root in the interval $\left(s_{0}, 1\right)$, provided $\eta<\eta_{c}$ where $\eta_{c}=\mu /\left[\sigma\left(1-\tau_{0}^{2}\right)^{1 / 2}\right]$. Again if $\eta=\eta_{c}$ we see that $s=1$ is a root of (20). Lastly, the said equation has no real root if $\eta>\eta_{c}$. Thus we have a critical wavelength, $L_{c}=2 \pi / \eta_{c}$, below which Rayleigh waves cannot propagate at all under the influence of surface stresses. The relation,

$$
(\mu / 2 \pi) L_{c}=\sigma\left(1-\left(b^{2} / a^{2}\right)\right)^{1 / 2},
$$

reveals that critical wavelength varies directly with residual surface tension which is a factor of surface stress.

\subsection{Love waves}

In order to consider Love waves we shall assume that the upper medium $\left(M_{2}\right)$ is of finite thickness $H$ and the lower medium $\left(M_{1}\right)$ is semi-infinite. Let the origin $O$ of the orthogonal cartesian frame $O x_{1} x_{2} x_{3}$ be on the interface, $O x_{1}$ and $O x_{3}$ are along the interface and vertically downwards into $M_{1}$ respectively. For Love wave propagation along $x_{1}$ axis (see figure 1),

$$
\begin{aligned}
& u_{1}=u_{3}=0, \\
& u_{2}=u_{2}\left(x_{1}, x_{3}, t\right) .
\end{aligned}
$$

Here equations of motion are

$$
\begin{aligned}
& \left(\nabla^{2}-\frac{1}{b^{2}} \frac{\partial^{2}}{\partial t^{2}}\right) u_{2}=0, \\
& \left(\nabla^{2}-\frac{1}{b^{\prime 2}} \frac{\partial^{2}}{\partial t^{2}}\right) u_{2}^{\prime}=0 .
\end{aligned}
$$

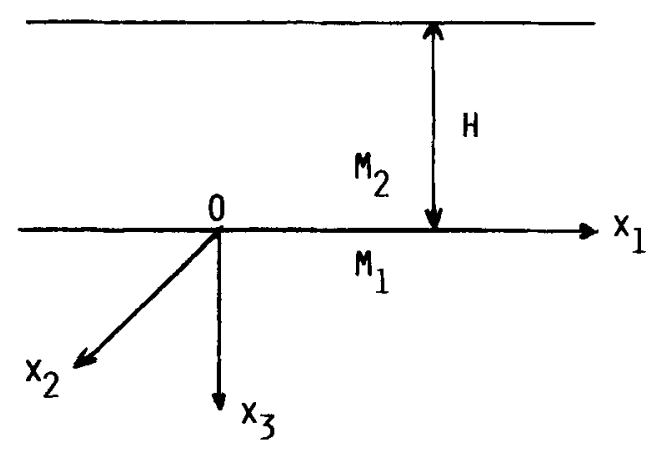

Figure 1. Love waves and their propagation. 
The solutions of (22) are

$$
\begin{aligned}
& u_{2}=A_{1} \exp \left(-\eta m_{2} x_{3}\right) \mathrm{e}^{i\left(\eta x_{1}-\zeta t\right)} \\
& u_{2}^{\prime}=\left\{A_{2} \exp \left(-\eta m_{2}^{\prime} x_{3}\right)+A_{3} \exp \left(\eta m_{2}^{\prime} x_{3}\right)\right\} \mathrm{e}^{i\left(\eta x_{1}-\zeta t\right)},
\end{aligned}
$$

where

$$
\begin{aligned}
m_{2} & =\left(1-s^{2}\right)^{1 / 2}, \\
m_{2}^{\prime} & =\left(1-s^{2}\right)^{1 / 2}, \\
C & =\frac{\zeta}{\eta}, \quad s=\frac{C}{b}, \quad s^{\prime}=\frac{C}{b^{\prime}} .
\end{aligned}
$$

The boundary conditions are:

(i) $u_{2}$ is continuous on the boundary surface of separation $x_{3}=0$;

(ii) $\tau_{23}$ is continuous on the boundary surface of separation $x_{3}=0$;

(iii) since the boundary surface $x_{3}=-H$ is free of external loads

we have (Gurtin \& Murdoch 1974-75)

$$
\tau_{23}^{\prime}+\sum_{2 \alpha, \alpha}=\rho_{0} \frac{\partial^{2} u_{2}^{\prime}}{\partial t^{2}} \quad \text { for } x_{3}=-H \text {. }
$$

Applying the above boundary conditions to (23) and then eliminating the constants $A_{1}$, $A_{2}, A_{3}$ we get the wave velocity equation for Love waves under the influence of surface stresses in determinantal form as,

$$
\left|\begin{array}{ccc}
1 & -1 & -1 \\
\mu m_{2} & -\mu^{\prime} m_{2}^{\prime} & \mu^{\prime} m_{2}^{\prime} \\
0\left\{\frac{\mu^{\prime} m_{2}^{\prime}}{\eta}+\left(\mu_{0}-\rho_{0} c^{2}\right)\right\} & \mathrm{e}^{\eta m_{2}^{\prime} H}
\end{array}-\left\{\frac{\mu^{\prime} m_{2}^{\prime}}{\eta}-\left(\mu_{0}-\rho_{0} c^{2}\right)\right\} \mathrm{e}^{-\eta m_{2}^{\prime} H}\right|=0
$$

This determinantal equation on simplification gives

$$
\begin{aligned}
\eta H= & \frac{1}{\left\{\left(c / b^{\prime}\right)^{2}-1\right\}^{1 / 2}} \\
& \quad \times \tan ^{-1}\left[\frac{\left\{\left(c^{2} / b^{2}\right)-1\right\}^{1 / 2}\left\{\left(\mu / \mu^{\prime}\right)\left[1-\left(c^{2} / b^{2}\right)\right]^{1 / 2}+\left(\eta / \mu^{\prime}\right)\left(\mu_{0}-\rho_{0} c^{2}\right)\right\}}{\left(c^{2} / b^{2}\right)-1-\left(\mu / \mu^{\prime}\right)\left[1-\left(c^{2} / b^{2}\right)\right]^{1 / 2} \cdot\left(\eta / \mu^{\prime}\right)\left(\mu_{0}-\rho_{0} c^{2}\right)}\right] .
\end{aligned}
$$

when the surface stress effect is neglected $\left(\mu_{0}=\rho_{0}=0\right),(25)$ reduces to

$$
\eta H=\frac{1}{\left\{\left(c^{2} / b^{\prime 2}\right)-1\right\}^{1 / 2}} \tan ^{-1}\left[\frac{\left(\mu^{\prime} / \mu\right)\left\{1-c^{2} / b^{2}\right\}^{1 / 2}}{\left\{\left(c^{2} / b^{2}\right)-1\right\}^{1 / 2}}\right],
$$

which is the classical Love wave velocity equation. From (25) we observe that Love wave propagation is possible if

$$
1<\left(c / b^{\prime}\right)<\left[(\mu / \rho) /\left(\mu^{\prime} / \rho^{\prime}\right)\right]^{1 / 2} .
$$


Table 1. Values of $\eta H$.

\begin{tabular}{llllllllll}
\hline$\eta$ & 1.048 & 1.096 & 1.144 & 1.192 & 1.240 & 1.288 & 1.336 & 1.384 & 1.432 \\
\hline 1 & 6.933 & 4.081 & 2.858 & 2.147 & 1.671 & 1.325 & 1.057 & 0.838 & 0.648 \\
2 & 7.444 & 4.576 & 3.333 & 2.602 & 2.104 & 1.733 & 1.439 & 1.193 & 0.973 \\
3 & 7.623 & 4.757 & 3.517 & 2.788 & 2.291 & 1.922 & 1.628 & 1.381 & 1.159 \\
$0^{*}$ & 2.975 & 1.630 & 1.078 & 0.771 & 0.571 & 0.428 & 0.317 & 0.225 & 0.139 \\
\hline
\end{tabular}

${ }^{*}$ Denotes the classical case where the surface stresses are not present

\subsection{Numerical calculation of Love waves}

For numerical calculations we take

$$
\begin{aligned}
& \mu=3.00 \times 10^{6} \mathrm{~N} / \mathrm{cm}^{2}, \quad \mu^{\prime}=5.00 \times 10^{6} \mathrm{~N} / \mathrm{cm}^{2}, \quad \mu_{0}=6.47 \times 10^{6} \mathrm{~N} / \mathrm{cm} \\
& \rho=2.72 \mathrm{~g} / \mathrm{cm}^{3}, \quad \rho^{\prime}=9.89 \mathrm{~g} / \mathrm{cm}^{3}, \quad \rho_{0}=3.40 \mathrm{~g} / \mathrm{cm}^{2}
\end{aligned}
$$

Table 1 gives the values of $\eta H$ for different values of $C / b^{\prime}$ taking $\eta=1,2,3$. It also gives the values of $\eta H$ for different values of $C / b^{\prime}$ in the absence of surface stresses (denoted by 0 in the first column).

We observe that the Love wave velocity equation is independent of $\sigma$ and depends solely upon $\mu_{0}$ and $\rho_{0}$. It is also observed that for a particular value of $\eta$, wave velocity decreases with increase of $\eta H$. Again for a particular wave velocity, $\eta H$ increases with increase of $\eta$. For comparison we give the values of $\eta H$ in table 1 for the classical case (denoted by 0 ) where the surface stresses are not present.

\subsection{Stoneley waves}

Stoneley waves are a generalized form of Rayleigh waves propagating along the common boundary of $M_{1}$ and $M_{2}$. Equation (17) represents the wave velocity equation for Stoneley waves in elastic media with surface stresses. This equation after a little manipulation may be obtained in the form

$$
\begin{aligned}
& \eta^{2} C D(1-S T)\left(1-S^{\prime} T^{\prime}\right) \\
& +s^{2}\left\{(C T+D S)\left(1-S^{\prime} T^{\prime}\right)+\frac{\mu^{\prime}}{\mu} \tau_{2}^{2}\left(C T^{\prime}+D S^{\prime}\right)(1-S T)\right\} \eta-\Delta(s)=0,
\end{aligned}
$$

where

$$
\begin{aligned}
\Delta(s)=(1- & \left.S^{\prime} T^{\prime}\right)\left\{\left(2-s^{2}\right)^{2}-4 S T-4 \frac{\mu^{\prime}}{\mu}\left(1-s^{2}-S T\right)\right\} \\
& +(1-S T)\left\{\left(2-\tau_{2}^{2} s^{2}\right)^{2}-4 S^{\prime} T^{\prime}-4 \frac{\mu^{\prime}}{\mu}-\left(1-\tau_{2}^{2} s^{2}-S^{\prime} T^{\prime}\right)\right\} \\
& -\tau_{2}^{2} s^{4} \frac{\mu^{\prime}}{\mu}\left(2+S T^{\prime}+S^{\prime} T\right),
\end{aligned}
$$


and

$$
\begin{aligned}
& s=c / b, \quad C=\sigma^{*} / \rho b^{2}, \quad \sigma^{*}=\sigma-\rho_{0} c^{2}, \\
& D=\frac{\Gamma^{*}}{\rho b^{2}}, \quad \Gamma^{*}=\Gamma-\rho_{0} c^{2}, \quad \Gamma=\lambda_{0}+2 \mu_{0}, \\
& S=\left(1-s^{2}\right)^{1 / 2}, \quad T=\left(1-\tau_{1}^{2} s^{2}\right)^{1 / 2}, \\
& S^{\prime}=\left(1-\tau_{2}^{2} s^{2}\right)^{1 / 2}, \quad T^{\prime}=\left(1-\tau_{3}^{2} s^{2}\right)^{1 / 2}, \\
& \tau_{1}=b / a, \quad \tau_{2}=b / b^{\prime}, \quad \tau_{3}=b / a^{\prime} .
\end{aligned}
$$

For the classical case, i.e., when surface stress is absent, we take $\sigma=\Gamma=\rho_{0}=0$, so that $C=D=0,(28)$ reduces to $\Delta(s)=0$ which is equivalent to the classical Stoneley wave velocity equation. It is observed that the Stoneley waves under the influence of surface stress are dispersive in contrast with the classical situation for which waves of all frequencies propagate uniformly at the same speed. The equation $\Delta(s)=0$ was solved numerically by Koppe (1948) who concluded that the velocity of the interface wave falls between the values of the velocity of Rayleigh waves and of the transverse waves in the medium with greater acoustic density. Taking $\mu^{\prime} \rightarrow 0$ in (28) we get the Rayleigh wave velocity equation under the influence of surface stress as

$$
\eta^{2} C D(1-S T)+s^{2}(C T+D S) \eta-g(s)=0,
$$

where

$$
g(s)=\left(2-s^{2}\right)-4 S T .
$$

This result is in agreement with the result obtained in (18) and also that by Murdoch (1976). Further, when there is no surface stress, this equation reduces to the classical Rayleigh wave velocity equation $g(s)=0$. This equation has one real root $s=s_{0}$, in the interval $(0,1)$. If $s=s_{1}$ satisfies the equation $\Delta(s)=0$, then $s_{0}<s_{1}<b \times$ transverse wave velocity in the medium with greater acoustical density. From (28) when $C D \neq 0$ we have

$$
\begin{aligned}
\eta=\{ & -s^{2}\left[(C T+D S)\left(1-S^{\prime} T^{\prime}\right)+\frac{\mu^{\prime}}{\mu} \tau_{2}^{2}\left(C T^{\prime}+D S^{\prime}\right)(1-S T)\right] \\
& \pm\left[s^{4}\left[(C T+D S)\left(1-S^{\prime} T^{\prime}\right)+\frac{\mu^{\prime}}{\mu} \tau_{2}^{2}\left(C T^{\prime}+D S^{\prime}\right)(1-S T)\right]^{2}\right. \\
& \left.\left.+4 \Delta(s) C D(1-S T)\left(1-S^{\prime} T^{\prime}\right)\right]^{1 / 2}\right\} \div\left\{2 C D(1-S T)\left(1-S^{\prime} T^{\prime}\right)\right\} .
\end{aligned}
$$

If $C=0$, i.e. in the absence of residual surface tension for a specific value of $s$ we have from (28)

$$
\eta=\Delta(s) /\left[s^{2}\left\{D S\left(1-S^{\prime} T^{\prime}\right)+\frac{\mu^{\prime}}{\mu} \tau_{2}^{2} D S^{\prime}(1-S T)\right\}\right] .
$$


Again when $D=0$ i.e. in the absence of surface tension at some value of $s$ we have

$$
\eta=\Delta(s) /\left[s^{2}\left\{C T\left(1-S^{\prime} T^{\prime}\right)+\frac{\mu^{\prime}}{\mu} \tau_{2}^{2} C T^{\prime}(1-S T)\right\}\right] .
$$

If both $C$ and $D$ vanish at some value $s^{\prime}$, we have $\Delta\left(s^{\prime}\right)=0$ so that Stoneley waves exist. Otherwise there is no propagation of Stoneley waves. The case when $\sigma, \Gamma>\rho_{0} b^{2}, C$ and $D$ will remain positive in $(0,1)$. Since $\eta$ is positive, $C<b, \tau_{1}, \tau_{2}, \tau_{3}<1$ we discard the solution of (34) which is negative for some $s$ in $(0,1)$. Thus we see that Stoneley wave velocity under the influence of surface stresses may be less than or greater than the classical Stoneley wave velocity but always remains greater than the Rayleigh wave velocity. Moreover, in the case when $0<\sigma<\Gamma<\rho_{0} b^{2}, C$ and $D$ will vanish exactly at one point (each on $(0,1)$ \}, say $s_{2}$ and $s_{3}$ on $(0,1)$, so that $s_{2}^{2}=\sigma / \rho_{0} b^{2}, s_{3}^{2}=\Gamma / \rho_{0} b^{2}$ and hence $0<s_{2}<s_{3}<1$, where $C$ is positive on $\left(0, s_{1}\right)$ and negative on $\left(s_{1}, 1\right)$ and $D$ is positive on $\left(O, s_{2}\right)$ and negative on $\left(s_{2}, 1\right)$ Again when $\sigma, \Gamma$ lie in $\left(0, \rho_{0} b^{2}\right), C T+D S$ and $C T^{\prime}+D S^{\prime}$ vanish at say $s_{4}$ and $s_{5}$ in $\left(s_{2}, s_{3}\right)$. We observe that $C T+D S$ is positive on $\left(0, s_{4}\right)$ and negative on $\left(s_{4}, 1\right)$ while $C T^{\prime}+D S^{\prime}$ is positive on $\left(0, s_{5}\right)$ and negative on $\left(s_{5}, 1\right)$. Thus the number of positive values of $\eta$ which satisfy $(28)$ for a particular value of $s$ depends upon the location of $s_{1}$ related to $s_{2}, s_{3}, s_{4}$, and $s_{5}$, as well as on the value of $Q(s)$ and zeros of $Q(s)$ where

$$
\begin{aligned}
Q(s)=s^{4}\left\{(C T+D S)\left(1-S^{\prime} T^{\prime}\right)\right. & +\frac{\mu^{\prime}}{\mu} \tau_{2}^{2}\left(C T^{\prime}+D S^{\prime}\right)(1-S T) \\
& \left.+4 \Delta(s) C D(1-S T)\left(1-S^{\prime} T^{\prime}\right)\right\} .
\end{aligned}
$$

Further discussion in the light of Murdoch (1976) is not pursued in this paper due to its complicated nature and cumbersome calculation.

\section{Conclusion}

Stoneley waves under the influence of surface stresses may be discussed in a manner similar to Rayleigh waves. It is seen that the propagation of Stoneley waves under the influence of surface stresses is not always possible. Under certain restricted conditions such a propagation is possible. Moreover, Stoneley wave velocity under the influence of surface stresses may be less or greater than the classical Stoneley wave velocity, but always remains greater than the Rayleigh wave velocity. We point out here that Stoneley waves are dispersive in character in contrast with the classical situation. Rayleigh waves have been deduced as a particular case of Stoneley waves. In case of Love waves we have shown that the wave velocity equation does not depend on residual surface tension. Some numerical calculations highlight the effect of surface stresses on Love wave as described in the paper.

\section{References}

Bullen K E, Bolt 1985 An introduction to the theory of seismology, 4th edn (Cambridge: University Press) 
Chandrasekharaiah D S 1987 Effect of surface stresses and voids on Rayleigh waves in an elastic solid. Int. J. Eng. Sci. 25: 205-211

Chandrasekharaiah D S 1986 Surface waves in an elastic half-space with voids. Acta Mech. 62: $77-85$

Das S C, Acharya D P, Sengupta P R 1992 Surface waves in an inhomogeneous elastic medium under the influence of gravity. Mech. Appl. 37: 541-551

Dey S K, Sengupta P R 1978 Effects of anisotropy on surface waves under the influence of gravity Acta Geophys. Polon. 26: 291-298

Gurtin M E, Murdoch A I 1974-75 A continuum theory of elastic material surfaces. Arch. Rat. Mech. Anal. 57: 291-323

Gurtin M E, Murdoch A I 1976 Effect of surface stresses on wave propagation in solids. J. Appl. Phys. 47: 4414-4421

Koppe H 1948 Über Rayleigh-Wellen ander oberflächezweier Medien. Z. Angew. Math. Mech. 28: $355-360$

Murdoch A I 1976 The propagation of surface waves in bodies with material boundaries. J. Mech. Phys. Solids 24: 137-146

Pal P K, Acharya D P, Sengupta P R 1996 Effect of voids on the propagation of waves in an elastic layer. Sadhana 21: 477-485

Plaster H J 1972 Blast cleaning and allied processes (London: Industrial Newspapers) 